\title{
Validity of $t-\mathrm{J}$ approximation for extended Hubbard model with strong repulsion
}

\author{
V.O. Cheranovskii ${ }^{1}$, D.J. Klein ${ }^{2}$, E.V. Ezerskaya ${ }^{1}$, and V.V. Tokarev $^{1}$ \\ ${ }^{1}$ V.N. Karazin Kharkiv National University, 4 Svoboda Sq., Kharkiv 61022, Ukraine \\ E-mail: cheranovskii@i.ua \\ ${ }^{2}$ Texas A\&M University at Galveston, Galveston, TX, 77554 USA
}

Received June 1, 2017, published online September 25, 2017

\begin{abstract}
It is shown that for finite cyclic systems described by two band Hubbard Hamiltonian with strong electron repulsion the reduction to effective $t-J$ model may give incorrect description of the ground state symmetry due to neglect of the correlated hopping terms.

PACS: 75.10.Jm Quantized spin models, including quantum spin frustration;

75.40.Cx Static properties (order parameter, static susceptibility, heat capacities, critical exponents, etc.).
\end{abstract}

Keywords: Hubbard model, $t-J$ model, correlated hopping.

\section{Introduction}

One of the basic models in the theory of strongly correlated electron systems is so called $t-J$ model [1]. It can be derived from one-band Hubbard model with strong repulsion in second order of perturbation theory (PT) for the electron transfers allowing double occupancies in the limit of a small concentration of holes in half-filled band. If number of holes is comparable with number of electrons, the additional correlated hopping terms of PT expansion should be taken into account [2]. For linear Hubbard chain these hopping terms renormalized coupling parameter $J$ of the corresponding $t-J$ model only. For more complicated lattices like two-leg ladder or diamond chain similar renormalization cannot be done. Nevertheless, there is a big experience in successful application of $t-J$ model to the study of different correlated systems, for instance, in the theory of high- $T_{C}$ superconductivity [1,3-5] and people believe that this model reproduces adequately significant part of physics even at intermediate concentration of holes. In this work we study the effect of correlated hopping terms, omitting in $t-J$ model, on the lowest states of twoband Hubbard model for finite lattice fragments with the periodic boundaries (cycles). We show the importance of these terms for the correct description of the model ground state and lowest excitations.

\section{Two band 1D Hubbard model with strong repulsion}

Let us consider the Hubbard model defined on the linear chain formed by $N$ unit cells with two different types of sites " $a$ " and " $b$ " (Fig. 1). The corresponding Hamiltonian has the form

$$
\begin{aligned}
& \mathbf{H}=t_{a b} \sum_{i, j, \sigma}\left(\mathbf{a}_{i, \sigma}^{+} \mathbf{b}_{i, \sigma}+\mathbf{a}_{i+1, \sigma}^{+} \mathbf{b}_{i, \sigma}+\text { H.c. }\right)+\Delta \sum_{i, \sigma} \mathbf{a}_{i, \sigma}^{+} \mathbf{a}_{i, \sigma}+ \\
& +U_{a} \sum_{i, \sigma} \mathbf{a}_{i, \sigma}^{+} \mathbf{a}_{i, \sigma} \mathbf{a}_{i,-\sigma}^{+} \mathbf{a}_{i,-\sigma}+U_{b} \sum_{i, \sigma} \mathbf{b}_{i, \sigma}^{+} \mathbf{b}_{i, \sigma} \mathbf{b}_{i,-\sigma}^{+} \mathbf{b}_{i,-\sigma} .
\end{aligned}
$$

Here $t_{a b}$ is the hopping integral, describing the electron transfer between neighbor sites of the chain; $\Delta$ is a difference between the orbital energies for " $a$ " and " $b$ " sites; $U_{a}, U_{b}$ are Hubbard energies for " $a$ " and " $b$ " sites, respectively.

Well known realization of this model is, so called 1D Emery model for high- $T_{C}$ superconducting copper oxides [3-7] (in this case, instead of electrons, we should consider holes in copper and oxygen bands). Such a model may also describe the common electron system of some stacked donor-acceptor salts.

Let us suppose that total number of electrons equals to $N+1$ and model parameters satisfy the condition $U_{b}, \Delta \gg>t_{a b} \mid$. In this case each " $b$ " site is occupied by one electron and only one electron belongs to " $a$ " sites. In the limit $t_{a b} \rightarrow 0$ we have degenerate ground state of the chain with respect to the localization of electron on $a$ sites.

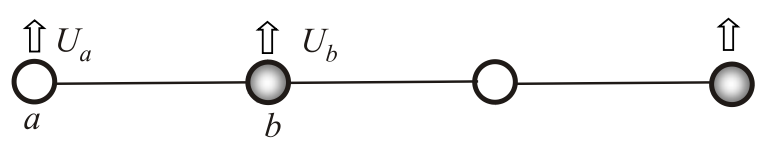

Fig. 1. Fragment of the chain system formed by two unit cells and described by the Hamiltonian (1). 
The doubly occupied sites have significantly bigger energy and effect on the lowest energy states of (1) in second order of PT in hopping integral $t_{a b}$. The corresponding second order PT processes result the translation of electron located on the given a site along the chain. First type of these processes is two consequent hops of " $a$ " and " $b$ " electrons, which may be depicted by following diagrams:

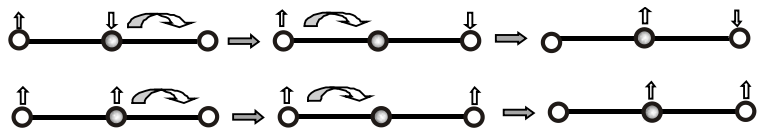

During these consequent hops the spin exchange is absent and there is no restriction on initial spin configuration of two electrons involved into the process.

For second processes the movement of the " $a$ " electron is performed through intermediate doubly occupied " $b$ ” site.

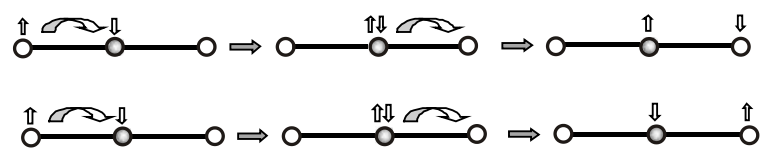

Therefore, such a movement is forbidden for parallel configurations of neighbor " $a$ " and " $b$ " electron spins. It corresponds to correlated hops of " $a$ " electron along the chain. These processes are absent in case $U_{b}=\infty$ studied in context of hole movement in 1D Emery model [4-7]. On the other side, the corresponding effective Hamiltonian for Emery model is essentially a $t-J$ model for the Hamiltonian (1). So, it is of interest to study the effect of the correlated hopping terms on the low-energy spectrum of initial Hamiltonian.

\section{Effective low-energy Hamiltonians}

Let enumerate all $N+1$ electron spins in succession over the chain sites independently of type. In second order of PT in $t_{a b}$ the Hamiltonian (1) for this chain can be written in the following form:

$$
\begin{aligned}
\mathbf{H}=\left(J_{1}+J_{2}\right) & {\left[\left(\mathbf{P}_{1,2}-1\right) \mathbf{c}_{1}^{+} \mathbf{c}_{1}+\sum_{n=2}^{N}\left(\mathbf{P}_{n-1, n}+\mathbf{P}_{n, n+1}-2\right) \mathbf{c}_{n}^{+} \mathbf{c}_{n}\right]+} \\
& +\sum_{n=1}^{N-1}\left[\left(J_{2} \mathbf{P}_{n, n+1}-J_{2}-J_{3}\right) \mathbf{c}_{n+1}^{+} \mathbf{c}_{n}+\text { H.c. }\right] .
\end{aligned}
$$

Here $\mathbf{c}_{n}^{+}$is a spinless Fermi operator which create electron on ith " $a$ " site, $\mathbf{P}_{n, m}$ is operator of the transposition of spin variables of two electrons with numbers $n$ and $m$

$$
J_{1}=\frac{\left(t_{a b}\right)^{2}}{\left(\Delta+U_{a}\right)}, \quad J_{2}=\frac{\left(t_{a b}\right)^{2}}{\left(U_{b}-\Delta\right)}, \quad J_{3}=\frac{\left(t_{a b}\right)^{2}}{\Delta} .
$$

In case of periodic boundary conditions this Hamiltonian should contain the additional term

$$
\begin{gathered}
\mathbf{H}_{1}=\mathbf{c}_{1}^{+} \mathbf{c}_{1}\left(J_{1}+J_{2}\right)\left(\mathbf{P}_{1, N+1}-1\right)+ \\
+(-1)^{N}\left\{\mathbf{c}_{N}^{+} \mathbf{c}_{1}\left[J_{2} \mathbf{Q}_{1, N}-\left(J_{2}+J_{3}\right) \mathbf{Q}_{1, N+1}\right]+\text { H.c. }\right\} .
\end{gathered}
$$

Here $\mathbf{Q}_{1, N+1}$ is the cyclic permutation operator of all spin variables $\sigma_{i}$ in spin part of wave function $\Phi\left(\sigma_{1}, \sigma_{2}, \sigma_{3}, \ldots \sigma_{N+1}\right)$.

$$
\mathbf{Q}_{1, N+1} \Phi\left(\sigma_{1}, \sigma_{2}, \sigma_{3}, \ldots \sigma_{N+1}\right)=\Phi\left(\sigma_{2}, \sigma_{3}, \ldots \sigma_{N+1}, \sigma_{1}\right)
$$

The Hamiltonian (2) has translation symmetry. Therefore, the eigenfunctions of (2) should be characterized by hole quasi-momentum $k=2 \pi l / N, \quad l=0,1,2 \ldots N-1$. The symmetry adapted basis functions corresponding to a fixed value of $k$ can be constructed by the combination of standard group theory approach and cyclic spin permutation technique $[8,9]$.

$$
\begin{gathered}
\Psi_{m, k}(S, M)=(N)^{-1 / 2} \sum_{n=1}^{N} \exp [i k(1-n)] \mathbf{Q}_{1, N+1}^{1-n} \mathbf{c}_{n}^{+}|0\rangle \Phi_{m}(S, M), \\
k=2 \pi l / N, \quad l=0,1 \ldots N-1
\end{gathered}
$$

Omitting simple but cumbersome manipulations with the cyclic permutations, we can rewrite the Hamiltonian (2) in the basis of functions (3) in the following form:

$$
\begin{gathered}
\mathbf{H}=\mathbf{H}_{t-J}+\mathbf{H}_{\mathrm{corr}}, \\
\mathbf{H}_{t-J}=\left(J_{1}+J_{2}\right)\left(\mathbf{P}_{1,2}+\mathbf{P}_{1, N+1}-2\right)+ \\
+J_{3}\left(\exp (i k) \mathbf{Q}_{1, N+1}+\text { H.c. }\right), \\
\mathbf{H}_{\text {corr }}=J_{2}\left[\exp (i k)\left(1-\mathbf{P}_{1, N+1}\right) \mathbf{Q}_{1, N+1}+\text { H.c. }\right] .
\end{gathered}
$$

(Here we used the unitary transformation $\mathbf{c}_{n}=(-1)^{n} \mathbf{c}_{n}$ in order to derive the same form of the effective low-energy Hamiltonian for even and odd $N$.)

The Hamiltonian $\mathbf{H}_{t-J}$ does not contain correlated hopping terms and corresponds to $t-J$ like model. For $J_{2}=0$ the Hamiltonian (5) coincides with the effective Hamiltonian for 1D Emery model with one hole in the oxygen band, which was introduced in [6,7]. The Hamiltonian (5) commutes with the operator of square of total spin $\mathbf{S}^{2}$ and the corresponding operator of $z$-projection of total spin $\mathbf{S}^{Z}$. Therefore the eigenstates of (5) can be classified by quantum numbers: total spin $S$ and $z$-projection of total spin $M$. The eigenvalue problem for (5) can be studied analytically for $S=(N \pm 1) / 2$. In particular, for the states with maximal value of $S$ there is simple analytical formula for the eigenvalues

$$
E_{k}=-2 J_{3} \cos (k) .
$$


The eigenvalue problem for the states with one inverted spin $(M=(N-1) / 2)$ can be reduced to finite difference equations studied in Appendix. The case of the states with more than one inverted spin is studied numerically only.

\section{Results of numerical simulation}

We study numerically the lowest energy states of the Hamiltonian (5) for finite lattice clusters with periodic boundaries containing 4-10 unit cells by means of Davidson method. Similar to [7] we put $U_{a}=0$, which corresponds to the condition $J_{1}=J_{3}$. The numerical calculations were performed in each subspace with given value of total spin $S$ and quasi-momentum $k$. The Hamiltonian matrix elements in subspace spanned on spin adapted basis functions were created by means of branching diagram technique [10]. The results of these calculations for the case $N=10$ are presented below on Figs. 2 and 3 (only the lowest energy levels with the total spin $S=(N+1) / 2-m$ and $0 \leq k \leq \pi$ are given).

We found numerically that the ground state is doubly degenerate and corresponds to $S=1 / 2, k= \pm 2 \pi / 5$. The lowest excited states with $S=3 / 2,5 / 2$ are also doubly degenerate. The increase of the value of $J_{2}$ leads to the change of the symmetry of above energy states (Fig. 3). For $J_{2}>4.33 J_{1}$ the ground state becomes nondegenerate and corresponds to $k=0$. For cluster formed by 8 unit cells the ground state corresponds to $k= \pm \pi / 2$ at $J_{1}=J_{2}=1$. The transition to the nondegenerate ground state with $k=0$ appear at $J_{2} \sim 2.874 J_{1}$. Probably, this effect is important only for finite clusters, because the increase of $N$ leads to more and more flat character of the lowest excitation bands.

In order to study the magnetic structure of the model ground state we calculated the values of the following spinspin correlator for the ground state $\Psi_{0}$ of the Hamiltonian (2):

$$
\rho=\left\langle\Psi_{0}\left|\sum_{n=1}^{N} \mathbf{S}_{n-1} \mathbf{S}_{n+1} \mathbf{c}_{n}^{+} \mathbf{c}_{n}\right| \Psi_{0}\right\rangle .
$$

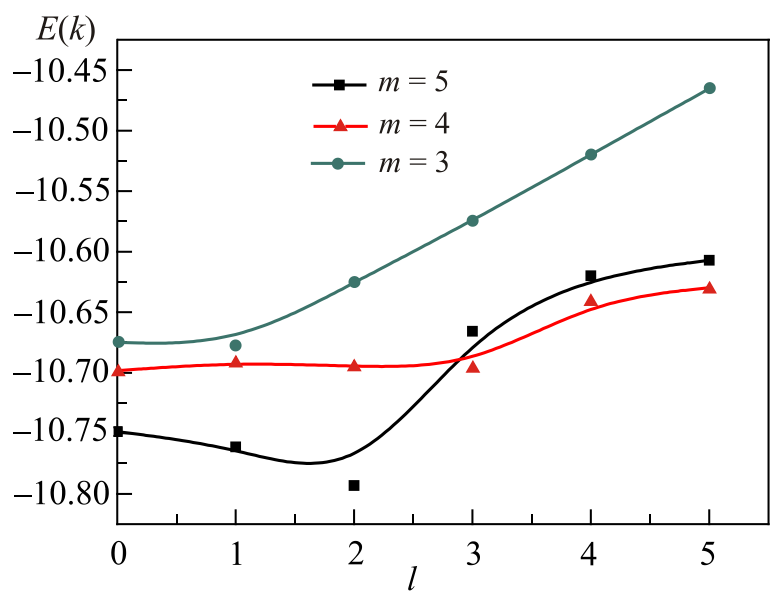

Fig. 2. (Color online) Lowest energy levels of given spin and symmetry for cyclic cluster with $N=10$ and $J_{1}=J_{2}=1, k=\pi l / 5$.

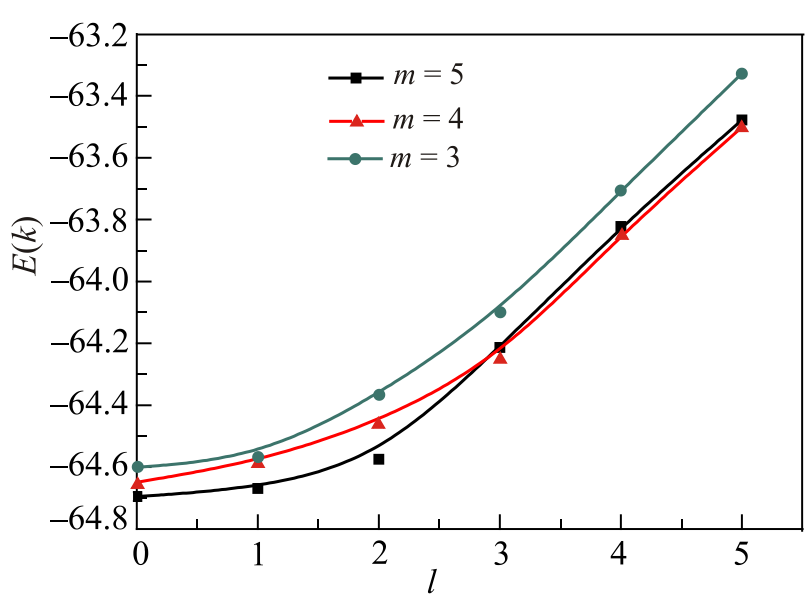

Fig. 3. (Color online) Lowest energy levels of given spin and symmetry for cluster with $N=10$ and $J_{1}=1, J_{2}=10, k=\pi l / 5$.

This quantity describes the ordering of " $b$ " spins in vicinity of the " $a$ " spin. It can be shown by spin permutation formalism, that the correlator (6) for the ground state $\Phi_{0}$ of the Hamiltonian (5) can be rewritten as

$$
\rho=\left\langle\Phi_{0}\left|\mathbf{S}_{2} \mathbf{S}_{N+1}\right| \Phi_{0}\right\rangle .
$$

The numerical calculations for cluster with $N=10$ showed preferably ferromagnetic character of the ordering of " $b$ " spins near to " $a$ " spin: $\rho \sim 0.2459$ for $J_{2}=J_{1}$ and $\rho \sim 0.2499$ for $J_{2}=10 J_{1}$.

If similar to $t-J$ model, we neglect the contribution of correlated hopping terms $\mathbf{H}_{\text {corr }}$ in model Hamiltonian (5), the transition to nondegenerate ground state is absent. The results of the corresponding calculations are presented in Fig. 4. The corresponding dispersion law is similar to the case $J_{1}=J_{2}=1$.

In the result we may conclude that for finite cyclic systems described by two band Hubbard Hamiltonian with strong electron repulsion the reduction to effective $t-J$ model may give incorrect description of the ground state symmetry due to neglect of the correlated hopping terms.

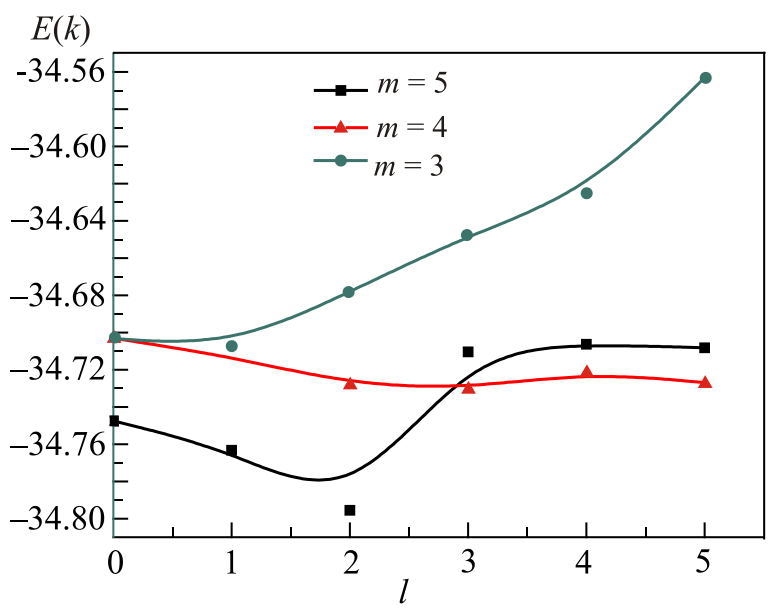

Fig. 4. (Color online) Lowest energy levels of given spin and symmetry for cluster with $N=10$ and $J_{1}=1, J_{2}=10$ in the absence of correlated hopping terms. 


\section{Acknowledgments}

VOC acknowledges the support of the VolkswagenStiftung, Germany (via grant 151110).

DJK acknowledges the support of the Welch Foundation of Houston, Texas (via grant BD-0894).

\section{Appendix. Exact solution for the states with one inverted spin}

The stationary states with $N+1$ electrons and one inverted spin are determined by Schrödinger equation (for simplicity we shift all the eigenvalues by constant $\left.2\left(J_{1}+J_{2}\right)\right)$

$$
\left(\mathbf{H}_{t-J}+\mathbf{H}_{\text {corr }}+2\left(J_{1}+J_{2}\right)\right)|\psi\rangle=\varepsilon|\psi\rangle,
$$

$$
|\psi\rangle=\sum_{m=1}^{N+1} A_{m} S_{m}^{-}|0\rangle .
$$

Here is $|0\rangle$ "vacuum" state, $|\psi\rangle$ is the vector of state. The wave function in the lattice-site representation $A_{m}$ obeys the finite difference equations

$$
\begin{gathered}
{\left[\varepsilon-2\left(J_{1}+J_{2}\right)\right] A_{m}-J_{3}\left(\mathrm{e}^{i k} A_{m-1}+\mathrm{e}^{-i k} A_{m+1}\right)=0,} \\
m \neq 1,2, N+1
\end{gathered}
$$

with the following "boundary conditions":

$$
\begin{gathered}
{\left[\varepsilon+2 J_{2} \cos k\right] A_{1}-\left[J_{1}+J_{2}+\left(J_{2}+J_{3}\right) \mathrm{e}^{-i k}\right] A_{2}-\left[J_{1}+J_{2}+\left(J_{2}+J_{3}\right) \mathrm{e}^{i k}\right] A_{N+1}=0} \\
{\left[\varepsilon-\left(J_{1}+J_{2}\right)\right] A_{1}-\left[J_{1}+J_{2}+\left(J_{2}+J_{3}\right) \mathrm{e}^{i k}\right] A_{1}-J_{3} \mathrm{e}^{-i k} A_{3}+J_{2} \mathrm{e}^{i k} A_{N+1}=0} \\
{\left[\varepsilon-\left(J_{1}+J_{2}\right)\right] A_{N+1}-\left[J_{1}+J_{2}+\left(J_{2}+J_{3}\right) \mathrm{e}^{-i k}\right] A_{1}-J_{3} \mathrm{e}^{i k} A_{N}+J_{2} \mathrm{e}^{-i k} A_{2}=0}
\end{gathered}
$$

Let seek the wave function in the form

$$
A_{m}=\mathrm{e}^{i k m}\left(C_{1} x^{m}+C_{2} x^{-m}\right), \quad m=2, \ldots, N+1 .
$$

Omitting simple but cumbersome calculation, we obtain from (A.2) the following dispersion equation:

$$
\varepsilon=2\left(J_{1}+J_{2}\right)-J_{3}\left(x+\frac{1}{x}\right),
$$

where parameter $x$ obeys the algebraic equation

$$
\begin{gathered}
u\left\{\left\{\left(\varepsilon_{1}-1-\beta\right)\left[u\left(\varepsilon_{1}-1-\beta\right)-2|r|^{2}\right]+\beta[p-\beta u]\right\} \sum_{m=0}^{N-2} x^{2 m}+\alpha^{2} u \sum_{m=0}^{N-4} x^{2(m+1)}-\right. \\
\left.-2 \alpha\left[u\left(\varepsilon_{1}-1-\beta\right)-|r|^{2}\right] \sum_{m=0}^{N-3} x^{2 m+1}+\alpha(2 \beta u-p) x^{N-2}\right\}=0 \\
\varepsilon_{1}=\varepsilon / J_{1} ; \quad u=\varepsilon_{1}+2 \beta \cos k ; \quad r=1+\beta+(\alpha+\beta) \mathrm{e}^{i k} \\
p=2 \operatorname{Re}(r) ; \quad \alpha=J_{3} / J_{1} ; \quad \beta=J_{2} / J_{1} .
\end{gathered}
$$

The values of $x=\exp (i q)$ correspond to quasicontinuous band and the real values of $x,|x|<1$ correspond to local levels.

The formula (A.3) and Eq. (A.4) reproduce the results of exact diagonalization study for the energy states of finite lattice clusters with $S=(N \pm 1) / 2$.

1. Yu.A. Izyumov, Phys. Usp. 34, 935 (1991).

2. D.J. Klein and W.A. Seitz, Phys. Rev. B 10, 3217 (1974).

3. V.J. Emery, Phys. Rev. Lett. 58, 2794 (1987).

4. L.I. Glazman and A.S. Ioselevich, JETP Lett. 47, 457 (1988).

5. M.W. Long, J. Phys. Condens. Matter 1, 9421 (1989).
6. V.Ya. Krivnov, A.A. Ovchinnikov, and V.O. Cheranovskii, Research Reports in Physics. Electron-Electron Correlation Effects in Low-Dimensional Conductors and Superconductors, Springer-Verlag, Heidelberg (1991), p. 86.

7. V.Ya. Krivnov and V.O. Cheranovskii, Fizika Tverdogo Tela 34, 3101 (1992).

8. V.O. Cheranovskii, Int. J. Quant. Chem. 41, 695 (1992).

9. V.O. Cheranovskii, O. Esenturk, and H.O. Pamuk, Phys. Rev. B 58, 12260 (1998).

10. V.O. Cheranovskii, Theoretical and Experimental Chemistry 20, 438 (1984). 